\title{
Análisis exploratorio de la relación entre ansiedad e inteligencia emocional de estudiantes universitarios cuidadores y no cuidadores durante la pandemia de Coronavirus. Caso: Cuenca, Ecuador
}

\author{
Exploratory analysis of the relationship between anxiety and emotional intelligence of \\ university students who are caregivers and non-caregivers during the coronavirus pandemic. \\ Case: Cuenca, Ecuador
}

\section{Kattya-Mineska Guerrero-Jiménez ${ }^{*}$ iD , Jimmy-Mauricio Pacheco-Ortega, Denisse-Micaela Romero-Cisneros, René-David Tacuri-Reino}

Universidad de Cuenca, Facultad de Psicología

* Autor de correspondencia: kattya.guerreroj@ucuenca.edu.ec

Fecha de recepción: 21 de noviembre de 2020 - Fecha de aceptación: 13 de abril de 2021

\begin{abstract}
RESUMEN
La investigación examinó la relación entre la inteligencia emocional y ansiedad en estudiantes universitarios cuidadores y no cuidadores durante la pandemia de coronavirus. Varios estudios señalan la aparición de factores de riesgo para la salud mental, como la ansiedad, durante una emergencia sanitaria. Si bien la inteligencia emocional podría ayudar como un factor protección, aún no está claro de si este y otros factores se diferencian cuando las personas actúan como cuidadores. Los participantes en este estudio fueron 60 estudiantes universitarios: 28 cuidadores y 32 no cuidadores. La investigación tuvo un diseño descriptivo y transversal no experimental, y utilizó la escala de inteligencia emocional de Wong y Law (WLEIS) y la escala de Ansiedad de Hamilton como instrumentos para medir la relación entre ansiedad e inteligencia emocional. Los resultados mostraron que no existen diferencias significativas entre el grupo de cuidadores y no cuidadores, mientras que se encontró una correlación significativa entre la ansiedad y los niveles de inteligencia emocional, niveles más altos de inteligencia emocional producen niveles más bajos de ansiedad psíquica. Los resultados del estudio exploratorio sugieren incluir en futuras investigaciones variables como la resiliencia y la incertidumbre.
\end{abstract}

Palabras clave: Inteligencia emocional, ansiedad, cuidadores, estudiantes no cuidadores, escala de ansiedad de Hamilton, escala de inteligencia emocional de Wong y Law (WLEIS).

\begin{abstract}
The research examined the relationship between emotional intelligence and anxiety in caregiver and non-caregiver college students during the corona pandemic. Several studies indicate the emergence of mental health risk factors, such as anxiety, during a health emergency. Whereas emotional intelligence could help as a protective factor, it is a question of whether this and other factors differentiate when people act as caregiver. Sixty college students, respectively 28 caregivers and 32 noncaregivers, participated in this study. The research had a descriptive and cross-sectional non-experimental design and used the Wong and Law' Emotional Intelligence Scale (WLEIS) and the Hamilton Anxiety scale as instruments for measuring the relationship between anxiety and emotional intelligence. The results showed that no significant differences exist between the group of caregivers and non-caregivers while a significant correlation was found between anxiety and emotional intelligence levels; higher levels of emotional intelligence produce lower levels of psychic anxiety. The findings of this explorative study suggest considering in future research variables such as resilience and uncertainty.
\end{abstract}

Keywords: Emotional intelligence, anxiety, caregiving students, non-caregiving students, Hamilton anxiety scale, Wong and Law emotional intelligence Scale (WLEIS).

\section{INTRODUCCIÓN}

Después de la declaratoria como pandemia en enero de 2020 debido al COVID-19 (OMS, 2020a), varios países tomaron medidas sanitarias como la suspensión temporal de actividades de todo tipo, como el cierre de fronteras y el aislamiento. Frente a la preocupación por las alertas del peligro que implicaba una enfermedad con alto riesgo de contagio y el incremento diario de las tasas de mortalidad, la preocupación de las personas en general pudo verse afectada. De hecho, este fenómeno es considerado como uno de los mayores problemas de salud pública internacional de las últimas décadas (OMS, 2020b; Sohrabi et al., 2020). 
La posibilidad de contagio, las posibles consecuencias tanto personales como familiares y la falta de inmunidad son las principales razones para que la salud mental de las personas pueda verse afectada (Faro et al., 2020). Por ejemplo, en ambientes hospitalarios, en donde el riesgo de contagio es más elevado, se ha podido evidenciar experiencias como exposiciones del temor a las consecuencias, sentimientos de soledad, negación, ansiedad, depresión, insomnio y desesperación; como consecuencia han aparecido mayores riesgos de suicidio y agresión, además de rasgos obsesivos compulsivos como controles repetitivos de temperatura y esterilización ( $\mathrm{Li}$ et al., 2020; Mamun \& Griffiths, 2020). En tal sentido, la preocupación y las conductas preventivas contra el posible contagio ha ido en aumento, mostrando que el riesgo, miedo y vulnerabilidad percibida sean bastante frecuentes. Yildırım et al. (2020) consideran que estas características traerían repercusiones en la salud mental, especialmente en la percepción subjetiva de la salud propia, angustia y satisfacción con la vida. Asimismo, Xiao (2020) sostiene que la salud mental estaría trastocada con depresión, miedo, ansiedad, aburrimiento, preocupación, tristeza, inseguridad, soledad e impotencia.

Aunque la preocupación sea un proceso cognitivo latente de todos los días, las condiciones por la pandemia podrían haber afectado la frecuencia y la intensidad de sentirse preocupados no solo acerca de la salud sino también por la situación económica, social y laboral. Las continuas incertidumbres orientadas hacia el peligro pueden hacer que las personas generen atajos mentales o creencias rígidas como formas de resolver los problemas, es decir, parecería que se genera un ciclo repetitivo en donde "estar preocupado" sería la solución para resolver "aquello que preocupa" y esto podría llegar a generar estados de ansiedad y depresión, orientación negativa al problema y conductas de evitación (González-Rodríguez et al., 2006 ; Mathews, 1990).

De hecho, las continuas limitaciones y miedos durante esta pandemia podrían generar respuestas psicológicas negativas que se relacionarían con Trastorno de Ansiedad Generalizada (TAG). González Rodríguez et al., (2006) identificaron procesos cognitivos, comportamentales, afectivos y fisiológicos vinculados con la preocupación patológica característica del TAG. En efecto, dichos procesos se refieren a la intolerancia a la incertidumbre, la evitación cognitiva, la orientación negativa al problema y la sobreestimación de la preocupación.

$\mathrm{Si}$ bien se han señalado varias manifestaciones de riesgo para la salud mental, esta investigación se centra en analizar la ansiedad, debido a que puede ser la principal causante de trastornos mentales, conductuales y psicofísicos (Rovella \& González-Rodríguez, 2008). Siguiendo al manual diagnóstico del DSM-V (American Psychiatric Association, 2014) la ansiedad se caracteriza como sentimiento de miedo exagerado e inapropiado ante eventos u objetos generales o específicos. Del mismo modo, el International Classification of Diseases for Mortality and Morbidity Statistics-11, categoriza como desorden de ansiedad generalizada a los síntomas como preocupación excesiva centrada en acontecimientos como finanzas, salud y trabajo, que persiste a lo largo del tiempo (ICD-11, 2018). En síntesis, la ansiedad es una respuesta emocional que se presenta ante situaciones amenazantes o peligrosas, generalmente calificada como patológica cuando es excesiva y frecuente (Rodríguez et al., 2014), por lo cual resulta pertinente analizar la presencia o ausencia de ansiedad durante la pandemia.

Por otra parte, es indispensable también considerar los factores de protección de salud mental como la inteligencia emocional (IE) puesto que es un concepto considerado como una herramienta útil para mejorar la calidad de vida y el desempeño de las personas (Colomeischi, 2015). Guevara (2011) menciona que el concepto de IE ha ido evolucionando a lo largo del tiempo. Charles Darwin planteó la importancia de la inteligencia como concepto de supervivencia y adaptación (MaturanaRomesin \& Mpodozis, 2000). Thorndike (1920) describió el término IE haciendo referencia a la inteligencia social, para conceptualizar la habilidad de comprender y motivar las personas. De hecho, las aportaciones de Mayer et al. (2008) conceptualizan a la IE como parte de la inteligencia social, la cual permite controlar, discriminar y usar aquella información que recibimos tanto de nosotros como de los demás con la capacidad de guiar nuestro comportamiento (Brackett \& Salovey, 2006). La IE permite abordar así la dimensión emocional de la naturaleza humana, y obtener una mejor y más amplia explicación acerca de por qué la inteligencia general no predice del éxito personal y social (Fernández-Berrocal \& Extremera-Pacheco, 2005). En este sentido, este constructo de Mayer et al. (2008) es bastante explicativo para describir y analizar las variables de esta investigación. Este modelo es definido desde distintas posturas teóricas, propone cuatro habilidades básicas para conceptualizar la IE. Estas cuatro habilidades se explican a continuación y son consecutivas:

- La "habilidad de percibir, valorar y expresar emociones con exactitud", qué implica identificar y reconocer tanto los propios sentimientos como aquellos de los que nos rodean, prestar atención y descodificar las señales emocionales de la expresión facial, tono de voz y movimientos corporales.

- La "habilidad de acceder y/o generar sentimientos que faciliten el pensamiento", que implica tener en cuenta los sentimientos cuando razonamos o resolvemos problemas, se centra en cómo las emociones afectan al sistema cognitivo y cómo los estados afectivos ayudan a tomar decisiones, ayudan también a priorizar los procesos cognitivos básicos, focalizando la atención en lo que es importante.

- La "habilidad de comprensión emocional", que implica desglosar las señales emocionales, etiquetar emociones y reconocer las categorías en que agrupan los sentimientos, implica una actividad anticipatoria como retrospectiva para conocer las causas generadoras del estado anímico y futuras consecuencias de las acciones que tomamos.

- Y, la "habilidad de regular emociones", que incluye la capacidad para estar abierto a los sentimientos positivos y negativos, reflexionar sobre ellos con el fin de descartar o aprovechar la información que los acompaña en función de su utilidad, implica la habilidad de regular propias emociones y también las ajenas, moderando las emociones negativas y cambiándolas por positivas, siendo esta habilidad la más compleja ya que alcanzaría los procesos emocionales más altos (Mayer et al., 2016).

De acuerdo, con los autores "al manejar los propios sentimientos, las personas deben ser capaces de observar, distinguir y etiquetar sus sentimientos de manera precisa, creer que pueden mejorarlos o modificarlos, hacer uso de 
estrategias que los cambiarán y valorar la eficacia de dichas estrategias" (Brackett \& Salovey, 2006). En esta medida, una de las emociones que es susceptible a la IE es la ansiedad, misma que es experimentada con malestar por el individuo, ya que generalmente se presenta ante situaciones estresantes, amenazantes o desconocidas, que el individuo percibe. Siendo así, la IE es un concepto que puede aplicarse a diferentes ámbitos de la vida de las personas, entre estos puede ser destacado el ámbito académico, en relación con la ansiedad en estudiantes universitarios cuidadores y no cuidadores.

Debido a que la pandemia ha ocasionado muchos cambios en la vida de las personas, en esta investigación fue también novedoso analizar la existencia o no de diferencias entre personas consideradas como cuidadoras y aquellas que no eran cuidadoras. Siguiendo a CoteloMosqueria (2015) se diferencian entre dos tipos de cuidadores, formales e informales. Los primeros hacen referencia a aquellos que atienden a terceros, ya sea debido a un deterioro funcional o cognitivo, quienes tienen una formación adecuada y reciben un salario por ejercer el trabajo. Mientras que los segundos son aquellos que ejercen la labor de cuidar de forma directa, sin formación y que no reciben un salario por realizar esta actividad, lo que incluye conyugues, familiares, amigos y vecinos (Cerquera \& Galvis Aparicio, 2014; Rogers \& Jarrott, 2008)

A partir de estas consideraciones, el objetivo general de esta investigación fue describir y relacionar los niveles de ansiedad e IE en estudiantes universitarios en condición de cuidadores y no cuidadores de la ciudad de Cuenca. En tal sentido, resultó novedoso para esta investigación describir si existen diferencias o no en los niveles de ansiedad o inteligencia emocional en cuidadores, debido a que, al tener como responsabilidad el cuidado por alguien más, se asumiría la hipótesis de que los cuidadores tendrán mayores niveles de ansiedad y también mayores niveles de inteligencia emocional. De hecho, se consideró a cuidadores informales o no cuidadores debido a que cada uno de ellos ejerce al mismo tiempo el rol de estudiante universitario de forma activa en universidades de la ciudad de Cuenca.

\section{MATERIALES Y MÉTODO}

La presente investigación fue de tipo descriptivo transversal con enfoque cuantitativo. Se pretendió relacionar los niveles de ansiedad e IE de estudiantes cuidadores y no cuidadores de universidades de Cuenca en función de diferentes variables y se desarrollará durante una situación puntual que es la del confinamiento debido al COVID-19 que actualmente vive la población.

\subsection{Participantes}

La muestra estuvo constituida por $n=60$ participantes $(45$ mujeres y 15 hombres), de los cuales 28 tuvieron la condición de cuidador y 32 no cuidadores con una edad media de 20.05 años y desviación estándar de 2.487 . Todos fueron estudiantes de diferentes universidades, en donde constan 41 participantes, 31 hombres y 10 mujeres, de la universidad pública Universidad de Cuenca; 6 participantes, 4 hombres y 2 mujeres, de la universidad privada Universidad del Azuay; 6 participantes, 4 hombres y 2 mujeres, de la universidad privada Universidad Católica de Cuenca; 5 participantes, 5 hombres y 0 mujeres, de la universidad pública Universidad Nacional de Educación; y 2 participantes, 1 hombre y 1 mujer de la universidad privada Universidad Politécnica Salesiana (de la ciudad de Cuenca). La evaluación se efectuó por una única vez durante el periodo de pandemia en el mes de junio y julio de 2020. Para la selección de los participantes se realizó un muestreo no probabilístico por conveniencia, que luego de aceptar voluntariamente la participación, cumplieron los criterios de inclusión y exclusión.

Los criterios de inclusión fueron: estudiantes con matrícula vigente en cualquier universidad de la ciudad de Cuenca, estudiantes en condición de cuidador y estudiante en condición de no cuidador, no hubo restricción en cuanto al sexo ni la edad. Mientras que los criterios de exclusión fueron: individuos sin una matrícula vigente en alguna de las universidades de la ciudad de Cuenca y quienes hayan expresado la condición de no desear participar.

\subsection{Tareas}

La escala de inteligencia emocional WLEIS (Law et al., 2004) es un cuestionario de tipo autoinforme que mide la inteligencia emocional como un tributo estable de una persona. Esta escala contiene 4 dimensiones y 4 ítems por dimensión. Usa un formato de respuesta ordinal de 7 puntos (desde 1; completamente en desacuerdo, hasta 7 completamente de acuerdo). Las 4 subescalas o factores son (a) valoración y expresión de las emociones propias (EEP), (b) valoración y reconocimiento de las emociones en otros (REO), (c) regulación de las propias emociones (REM) y (d) uso de la emoción para facilitar el desempeño (UEM). El Alpha de Cronbach general fue de $\alpha=.95$ (alta); el de la evaluación de las propias emociones: $\alpha=.898$; evaluación de las emociones de los demás: $\alpha=.898$; regulación de las propias emociones: $\alpha=.908$; uso de las emociones: $\alpha=.908$.

La escala de ansiedad de Hamilton (Servicio Andaluz de Salud, 2019) mide la severidad y presencia de la ansiedad, consta de 14 ítems, contiene un número de síntomas que está calificado en una escala de 0 a 4 , y considera 4 como una puntación más grave. De esta versión del instrumento se pueden obtener dos puntuaciones que corresponden a ansiedad psíquica (ítems 1, 2, 3, 4, 5, 6) y a ansiedad somática (ítems $7,8,9,10,11,12$ y 13). No existen puntos de corte. Una mayor puntuación indica una mayor intensidad de la ansiedad. Es sensible a las variaciones a través del tiempo o tras recibir tratamiento. El Alpha de Cronbach general fue de $\alpha=.740$; el de ansiedad psíquica fue: $\alpha=.780$; y el de ansiedad somática: $\alpha=.799$.

\subsection{Procedimientos}

Previo a la recolección de información se hizo un análisis de contenido ( 2 expertos y 2 personas con similares características de la población de estudio de los ítems de cada cuestionario utilizado y posteriormente se hizo un pilotaje con pares similares) usando el instrumento completo mediante Google Forms. El formulario de Google consideró una sección de datos sociodemográficos, la escala de inteligencia emocional WLEIS y la escala de ansiedad de Hamilton. Cada persona se demoró en promedio cerca de 10 minutos en responder todo el formulario. Además, dentro del mismo formulario 
se integró la información de la investigación para que así la persona al momento de iniciar, pueda aceptar o declinar su participación.

\section{RESULTADOS}

Los datos se recolectaron mediante la aplicación de Google Forms y las variables se codificaron a través del paquete estadístico SPSS v.23. El análisis de información se realizó con un índice de confiabilidad del $95 \%$ y con un margen de error del .05 . Antes del análisis estadístico inferencial y considerando que la muestra es mayor a 50 participantes, se aplicó la prueba Kolmogorov-Smirnov. Esta prueba demostró una distribución normal con un $p>.05$ por lo que se utilizaron pruebas paramétricas. En este sentido y en línea con los objetivos planteados se usó la prueba $\rho$ (rho) de Spearman para correlacionar ansiedad e IE, y la prueba $U$ de Mann-Whitney para comparar grupos.

En función del primer objetivo específico orientado a correlacionar la inteligencia emocional y ansiedad, la prueba $\rho$ de Spearman indicó que existieron correlaciones significativas de todas las dimensiones de inteligencia emocional con la ansiedad psíquica $(p<.05)$, mientras que en cuanto a la ansiedad somática no existieron correlaciones de forma significativa con ninguna de las variables (ver la Tabla 1). Esta correlación expresa que mientras las dimensiones de inteligencia emocional tienden a crecer la ansiedad psíquica desciende. Por lo tanto, se confirmó la hipótesis planteada mostrando que existe una correlación negativa significativa entre la IE y Ansiedad Psíquica. Estableciendo que, si existe una relación negativa significativa entre los dos constructos psicológicos, es decir que, si una persona posee mayor nivel de inteligencia emocional, entonces ésta misma persona presentaría menores niveles de ansiedad.

En relación con el segundo objetivo de este estudio, que trata sobre diferenciar los niveles ansiedad de estudiantes cuidadores y no cuidadores, la prueba $U$ de Mann-Whitney para muestras independientes no mostró diferencias significativas en los niveles de ansiedad psíquica y los niveles de ansiedad somática con las dimensiones de IE entre estudiantes cuidadores y no cuidadores. En base a los resultados obtenidos $(\mathrm{U}=.923$ y $p>.05$ y $\mathrm{U}=.345$ y $p<.05$ para cuidadores y no cuidadores, respectivamente) se acepta la hipótesis nula que no existe diferencia significativa entre los niveles de inteligencia emocional y los dos tipos de ansiedad (psíquica y somática) de estudiantes cuidadores y no cuidadores. En otras palabras, tener o no la función de cuidador, no diferencia los niveles de inteligencia emocional ni de ansiedad.

\section{DISCUSIÓN}

Derivados del objetivo de relacionar los niveles de Ansiedad e Inteligencia Emocional (IE) en estudiantes en condición de cuidador y no cuidador, la hipótesis planteaba que no existe una correlación significativa entre la inteligencia emocional y la ansiedad. Los resultados de esta investigación indicaron que sí existe una correlación inversamente significativa entre la IE y la ansiedad, lo que quiere decir que entre mayor manejo de IE posea una persona, menor ansiedad presentaría. Estos resultados coinciden con los presentados por Rodríguez de Ávila et al. (2011) quienes presentaron una relación inversa entre la compresión emocional y la ansiedad-estado, y entre la compresión emocional y ansiedad-rasgo, lo que quiere decir que, a mayor comprensión emocional, menor ansiedad-estado y menor ansiedad-rasgo. Así mismo, encontraron una relación inversa entre regulación emocional y la ansiedad-estado, así como entre la regulación emocional y la ansiedad-rasgo, lo que quiere decir que a mayor regulación emocional menor ansiedadestado y ansiedad-rasgo y viceversa.

De igual manera, Guerrero-Barona et al. (2019) en su investigación demostraron la existencia de correlaciones negativas entre todas las dimensiones de autoconcepto y la ansiedad. La inteligencia emocional se relacionó con el autoconcepto físico y social. Asimismo, confirmaron también que el sexo no influye en el autoconcepto global, pero sí en la ansiedad. Sosa-Baltasar (2014) por su parte, reflejó resultados similares, encontrando relaciones negativas entre ansiedad-autoconcepto-edad y las dimensiones académicas, física, emocional y social, de dicho constructo en universitarios. Por lo tanto, a mayor regulación emocional, mayor autoconcepto, académico, emocional y físico. En otro estudio realizado a jóvenes universitarios por Bojórquez \& Moroyoqui (2020) se encontró una relación entre IE percibida y la ansiedad en jóvenes universitarios, además de encontrar una relación directa entre la ansiedad como estado, rasgo y la reparación emocional. Sin embargo, Pulido-Acosta \& Herrera-Clavero (2017) demostraron que los predictores de la ansiedad son la edad y el género, mientras que los predictores para la IE fueron la edad, cultura y género; además, ellos no encontraron una relación directa entre ansiedad e IE, es decir, ninguna actuó como predictora de otra.

Tabla 1. Correlaciones de Spearman en la muestra total $(n=60)$ entre las dimensiones de Inteligencia Emocional (EEP, REO, REM y UEM) con Ansiedad Psíquica y Somática.

\begin{tabular}{lcccccc}
\hline & EEP & REO & REM & UEM & A. Psíquica & A. Somática \\
\hline EEP & 1 & $.745^{* *}$ & $.774^{* *}$ & $.634^{* *}$ & $-.462^{* *}$ & $-.267^{*}$ \\
REO & $.745^{* *}$ & 1 & $.796^{* *}$ & $.773^{* *}$ & $-.487^{* *}$ & -.226 \\
REM & $.774^{* *}$ & $.796^{* *}$ & 1 & $.758^{* *}$ & $-.407^{* *}$ & -.185 \\
UEM & $.634^{* *}$ & $.773^{* *}$ & $.758^{* *}$ & 1 & $-.389^{* *}$ & -.143 \\
\hline
\end{tabular}

** La correlación es significativa en el nivel $p<.01$ (bilateral).

* La correlación es significativa en el nivel $p<.05$ (bilateral).

EEP: Evaluación de las Propias Emociones; REO: Evaluación de Emociones de los Demás; REM: Regulación de Emociones; UEM: Uso de las Emociones. 
Respecto al segundo objetivo sobre diferenciar los niveles de ansiedad e inteligencia emocional en estudiantes universitarios en condición de cuidadores y no cuidadores de las universidades evaluadas, se realizó la prueba $t$ de Student para muestras independientes, dando como resultado la inexistencia de una diferencia significativa entre estos dos grupos. Estudios previos relacionados únicamente toman en cuenta la condición de cuidadores formales, por lo que sería recomendable que nuevos estudios analicen diferencias que valoren la función de cuidadores formales de una manera más detallada, donde se especifique el tipo de compromiso con la persona a la cual se presta el servicio de cuidado.

En cuanto a los resultados de estudiantes no cuidadores, estos coinciden con los del estudio de Rodríguez de Ávila et al. (2011) donde se evidencia el índice de IE mediante el TMMS- $24^{1}$ y la ansiedad mediante el instrumento IDARE $^{2}$, versión en español del $\mathrm{STAI}^{3}$, en una población universitaria de 167 estudiantes, dando como resultado que el $71.3 \%$ de la muestra puntúa en el nivel adecuado de percepción emocional. En el apartado de la compresión emocional, la mayor parte de la muestra $(69.5 \%)$ reveló una adecuada comprensión emocional, mientras que un $70.1 \%$ manifestaron una adecuada regulación de emociones. Esto demuestra que la inteligencia emocional en estudiantes universitarios se manifiesta de manera similar a los resultados de la presente investigación.

Con relación a la población de cuidadores universitarios informales, existe casi nula bibliografía en esta población, encontrándose con estudios previos enfocados a cuidadores formales y la IE, destacando el estudio piloto: "Inteligencia emocional y cuidadores formales, burnout y satisfacción laboral" de Cerquera-Córdoba et al. (2016) quienes evidenciaron que la inteligencia emocional se muestra como un componente significativo para el bienestar del cuidador, incluso con la condición de informalidad (no cuidador).

\section{CONCLUSIONES}

Los niveles de ansiedad de inteligencia emocional en los estudiantes universitarios de la ciudad de Cuenca se correlacionan significativamente de manera negativa, es decir, a mayor inteligencia emocional, menores niveles de ansiedad y viceversa. Este hallazgo se aplica a ambos grupos de estudiantes, cuidadores y no cuidadores. Asimismo, se encontró que existen un mayor nivel de ansiedad a nivel psíquico que a nivel somático. Además, al diferenciar los niveles de ansiedad e inteligencia emocional, no se encontraron diferencias significativas. Es de esperar que una expansión de la investigación podría conducir al descubrimiento de nuevas formas de afrontamiento y resiliencia frente a las « nuevas realidades de la convivencia»).

Como limitaciones al estudio cabría mencionar, que el acceso a la muestra fue limitado debido a la emergencia sanitaria por el COVID-19, lo cual no permite generalizar los resultados obtenidos. Otra limitación del estudio hace referencia al hecho de haber ajustado los instrumentos utilizados al formato de Google Forms, y esto podría

\footnotetext{
${ }^{1}$ Trait Meta-Mood Scale (Escala de estimulación magnética transversal)
}

interferir de alguna forma en el método de completar el cuestionario. Por ello, se puede sugerir replicar el estudio aplicando otro tipo de técnicas de evaluación (autoinforme, registro observaciones, etc.) con la finalidad de verificar los resultados obtenidos con este trabajo.

\section{REFERENCIAS}

American Psychiatric Association. (2014). DSM-V (R). American Journal of Psychiatry, 991. https://doi.org/10.1176/appi.books.9780890425596.29 5735

Bojórquez, C., \& Moroyoqui, S. (2020). Relación entre inteligencia emocional percibida y ansiedad en estudiantes universitarios. Espacios, 41(2), 267-278.

Brackett, M. A., \& Salovey, P. (2006). Measuring emotional intelligence with the Mayer-SaloveryCaruso Emotional Intelligence Test (MSCEIT). Psicothema, 18(SUPPL.1), 34-41.

Cerquera-Córdoba, A., Matajira-Camacho, Y., \& PabónPoches, D. (2016). Caracterización de una muestra de cuidadores formales de pacientes con trastorno neurocognitivo mayor en Bucaramanga. Revista Virtual Universidad Católica Del Norte, 1(47), 4-19.

Cerquera, M., \& Galvis-Aparicio, M. (2014). Efectos de cuidar personas con Alzheimer: un estudio sobre cuidadores formales e informales. Pensamiento Psicológico, 12(1), 149-167. https://doi.org/10.11144/javerianacali.ppsi12-1.ecpa

Colomeischi, A. A. (2015). Teacher burnout in relation with their emotional intelligence and personality traits. Procedia - Social and Behavioral Sciences, 180, 10671073. https://doi.org/10.1016/j.sbspro.2015.02.207

Cotelo-Mosqueira, Mónica. (2015). Estudio piloto: Inteligencia emocional en cuidadores formales, burnout y satisfacción laboral [Universidade da Coruña]. https://ruc.udc.es/dspace/bitstream/handle/2183/14531/ CoteloMosqueira_Monica_TFM_ 2015.pdf? sequence $=2 \&$ isAllowed $=y$

Faro, A., Bahiano, M. de A., Nakano, T. de C., Reis, C., da Silva, B. F. P., \& Vitti, L. S. (2020). COVID-19 and mental health: The emergence of care. Estudos de Psicologia (Campinas), 37, 1-14. PUC de Campinas. https://doi.org/10.1590/1982-0275202037E200074

Fernández-Berrocal, P., \& Extremera-Pacheco, N. (2005). La inteligencia emocional y la educación de las emociones desde el modelo de Mayer y Salovey. Revista Interuniversitaria de Formación Del Profesorado, 19(3), 63-93.

González-Rodríguez, M., Cubas, R., Rovella, A. T., \& Darias-Herrera, M. (2006). Adaptación española de la escala de intolerancia hacia la incertidumbre: procesos cognitivos, ansiedad y depresión. Psicología y Salud, 16(2), 219-233.

Guerrero-Barona, E., Sánchez-Herrera, S., MorenoManso, J., Sosa-Baltasar, D., \& Durán-Vinagre, M. (2019). El autoconcepto y su relación con la

\footnotetext{
2 Inventario De Ansiedad Rasgo Estado

${ }^{3}$ StateTrait Anxiety Inventory
} 
inteligencia emocional y la ansiedad. Behavioral Psychology/Psicologia Conductual, 27(3), 455-476.

Guevara, L. (2011). La inteligencia emocional. Temas para la Educación, Revista Digital para Profesionales de la Enseñanza, 12, 1-12. Retrieved from https://www.feandalucia.ccoo.es/docu/p5sd7866.pdf

ICD-11. (2018). ICD-11 International classification of diseases for mortality and morbidity statistics. Journal of Chemical Information and Modeling, 53(9), 16891699. https://doi.org/10.1017/CBO9781107415324.004

Law, K. S., Wong, C. S., \& Song, L. J. (2004). The construct and criterion validity of emotional intelligence and its potential utility for management studies. Journal of Applied Psychology, 89(3), 483496. https://doi.org/10.1037/0021-9010.89.3.483

Li, W., Yang, Y., Liu, Z. H., Zhao, Y. J., Zhang, Q., Zhang, L., ... Xiang, Y. T. (2020). Progression of mental health services during the COVID-19 outbreak in China. International Journal of Biological Sciences, 16, 1732-1738. https://doi.org/10.7150/ijbs.45120

Mamun, M. A., \& Griffiths, M. D. (2020). First COVID19 suicide case in Bangladesh due to fear of COVID19 and xenophobia: Possible suicide prevention strategies. Asian Journal of Psychiatry, 51, 102073. https://doi.org/10.1016/j.ajp.2020.102073

Mathews, A. (1990). Why worry? The cognitive function of anxiety. Behaviour Research and Therapy, 28(6), 455-468. https://doi.org/10.1016/0005-7967(90)901323

Maturana-Romesin, H., \& Mpodozis, J. (2000). El origen de las especies por medio de la deriva natural. Revista chilena de historia natural, 73(2), 261-310. https://doi.org/10.4067/S0716-078X2000000200005

Mayer, J. D., Caruso, D. R., \& Salovey, P. (2016). The ability model of emotional intelligence: Principles and updates. Emotion Review, 8(4), 290-300. https://doi.org/10.1177/1754073916639667

Mayer, J. D., Salovey, P., \& Caruso, D. R. (2008). Emotional Intelligence: New ability or eclectic traits? American Psychologist, 63(6), 503-517. https://doi.org/10.1037/0003-066X.63.6.503

OMS. (2020a). Coronavirus disease 2019 (COVID-19): Situation report - 78. Geneva. https://apps.who.int/iris/handle/10665/331719

OMS. (2020b). Coronavirus disease 2019 (covid-19). Journal of Internal Medicine of Taiwan, 31(2), 61-66. https://doi.org/10.6314/JIMT.202004_31(2).01

Pulido-Acosta, F., \& Herrera-Clavero, F. (2017). La inteligencia emocional como predictora del rendimiento académico en la infancia: El contexto pluricultural de Ceuta. Revista Complutense de Educacion, 28(4), 1251-1265. https://doi.org/10.5209/RCED.51712

Rodríguez, A., Dapía, M. D., \& López-Castelo, A. (2014). Ansiedad ante los exámenes en alumnos de Educación Secundaria obligatoria. Revista de Estudios e Investigación en Psicología y Educación, 1(2), 132. https://doi.org/10.17979/reipe.2014.1.2.14

Rodríguez de Ávila, U., Amaya-Agudelo, A. M., \& Argota-Pineda, A. P. (2011). Inteligencia emocional y ansiedad en estudiantes universitarios. Psicogente, 14(26), 310-320.

Rogers, S. D., \& Jarrott, S. E. (2008). Cognitive impairment and effects on upper body strength of adults with dementia. Journal of Aging and Physical Activity, 16(1), 61-68. https://doi.org/10.1123/japa.16.1.61

Rovella, A., \& González-Rodríguez, M. (2008). Trastorno de ansiedad generalizada. Redalyc, 1, 179194.

Servicio Andaluz de Salud. (2019). Escala de ansiedad de Hamilton. Retrieved from http://www.hvn.es/enfermeria/ficheros/escala_de_ansie dad_de_hamilton.pdf

Sohrabi, C., Alsafi, Z., O’Neill, N., Khan, M., Kerwan, A., Al-Jabir, A., ... Agha, R. (2020). World Health Organization declares global emergency: A review of the 2019 novel coronavirus (COVID-19). International Journal of Surgery, 76, 71-76. https://doi.org/10.1016/j.ijsu.2020.02.034

Sosa-Baltasar, D. (2014). Relación entre autoconcepto, ansiedad e inteligencia emocional: eficacia de un programa de intervención en estudiantes adolescentes Conformidad de los directores.

https://doi.org/http://dehesa.unex.es/xmlui/bitstream/ha ndle/10662/1678/TDUEX_2014_Sosa_Baltasar.pdf?se quence $=1$

Thorndike, E. L. (1920). Intelligence and its uses. Harper's Magazine, 140, 227-235.

Xiao, C. (2020). A novel approach of consultation on 2019 novel coronavirus (COVID-19)-related psychological and mental problems: Structured letter therapy. Psychiatry Investigation, 17, 175-176. https://doi.org/10.30773/pi.2020.0047

Yıldırım, M., Geçer, E., \& Akgül, Ö. (2020). The impacts of vulnerability, perceived risk, and fear on preventive behaviours against COVID-19. Psychology, Health \& Medicine, 26(1), 35-43. https://doi.org/10.1080/13548506.2020.1776891 